\section{Atenção ao planejamento familiar no Brasil hoje: reflexões sobre os resultados de uma pesquisa}

\author{
Family planning in Brazil today: \\ an analysis of recent research
}

\begin{abstract}
This two-component study (descriptive crosssectional and qualitative) assessed the availability of contraceptives in primary care clinics in Brazilian municipalities. The family planning program was also analyzed as part of the country's Family Health Strategy. Phone interviews were held with local health managers to obtain information on contraceptive supply in a selected sample of municipalities. Four municipalities were selected and visited for the qualitative analysis, using direct observation and semi-structured interviews with health professionals and managers. Descriptive statistical and multiple logistic regression analyses were performed. Content analysis technique was used for qualitative data. According to the results, family planning activities are often not integrated with other health activities. Health professionals and managers failed to understand family planning as part of primary health care and felt unable to assist patients. Family planning in Brazil is marked by the unavailability of contraceptives in public health programs.
\end{abstract}

Family Planning (Public Health); Contraception; Primary Health Care; Family Health

\author{
Maria José Duarte Osis 1 \\ Aníbal Faúndes 1 \\ Maria Yolanda Makuch 1 \\ Maeve de Brito Mello ${ }^{1}$ \\ Maria Helena de Sousa 1 \\ Maria José de Oliveira Araújo 2
}

\section{Introdução}

Desde o lançamento do Programa de Assistência Integral à Saúde da Mulher (PAISM), em 1983, o governo brasileiro tem atuado nas questões relativas ao planejamento familiar, adotando políticas e medidas para permitir o acesso da população aos meios de contracepção. Os primeiros esforços para implementar o PAISM incluíram a provisão de insumos contraceptivos para os serviços públicos de saúde e a capacitação dos profissionais para proverem atenção em planejamento familiar com qualidade 1 . Entretanto, esses esforços foram marcados pela descontinuidade e baixa efetividade, e os avanços foram muito lentos na segunda metade dos anos de 1980 e quase que durante toda a década seguinte 1,2 .

Em outubro de 2001, foi proposta uma nova estratégia para aquisição de métodos anticoncepcionais reversíveis pelo Ministério da Saúde para os municípios que atendessem a determinados critérios 3 , com distribuição descentralizada e trimestral, visando a uma cobertura de aproximadamente $30 \%$ da demanda potencial por esses métodos no Sistema Único de Saúde (SUS). Por ocasião do envio da primeira remessa de insumos aos municípios, em julho de 2002, o Ministério da Saúde solicitou ao Centro de Pesquisas em Saúde Reprodutiva de Campinas (CEMICAMP) que realizasse uma pesquisa para verificar se os métodos anticoncepcio- 
nais enviados aos municípios estavam disponíveis nas Unidades Básicas de Saúde (UBS), e identificar possíveis obstáculos a essa disponibilidade. Neste artigo são apresentados os resultados desse estudo.

\section{Sujeitos e métodos}

Estudo com abordagem quantitativa e qualitativa 4,5: primeira etapa descritiva, de corte transversal, e segunda etapa de estudo de casos 6 .

Os métodos anticoncepcionais eram enviados aos municípios na forma de kits: (i) kit básico, composto pela pílula combinada, pílula só de progesterona (minipílula) e preservativo masculino; e (ii) kit complementar, composto pelo dispositivo intra-uterino (DIU) e pelo injetável trimestral, enviado somente aos municípios com população igual ou superior a 50 mil habitantes, ou que informavam ao Ministério da Saúde possuir profissionais capacitados para a inserção do DIU ${ }^{3}$. Para a primeira etapa da pesquisa, o tamanho da amostra foi definido para avaliar o recebimento de cada tipo de kit por Secretarias Municipais de Saúde (SMS) e UBS. Foi desenhado um plano complexo de amostragem, incluindo-se no estudo sobre o kit básico 499 SMS e 526 UBS, e 98 SMS e 125 UBS para o kit complementar.

Entre agosto e outubro de 2002, foram aplicados, por telefone, questionários sobre o recebimento recente de métodos anticoncepcionais, respondidos pelo(a) responsável pela então chamada Farmácia Popular, e pelo(a) gerente/coordenador(a) de UBS sorteadas em cada município.

Após o processamento dos dados, procedeu-se inicialmente a análise estatística descritiva 7 . As características do plano de amostragem - estrato, conglomerado e ponderação foram consideradas na análise para evitar subestimativas dos erros de amostragem 8,9 . Foram calculadas as proporções usando-se o estimador razão, intervalos de confiança de 95\% para as mesmas e efeito do desenho (Deff), que se refere à razão da variância segundo o plano complexo de amostragem utilizado, dividido pela variância considerando-se a amostra como se fosse AAS; a variância foi estimada utilizando-se aproximação de Taylor 8 . O programa utilizado para a análise dos dados foi o Epi Info 6.04b (Centers for Disease Control and Prevention, Atlanta, Estados Unidos).

Em uma segunda etapa, avaliou-se a relação entre a situação de cada município e o recebimento dos métodos anticoncepcionais utilizando como indicadores aspectos sociais, de- mográficos e do atendimento à saúde. Procedeu-se à análise de regressão logística múltipla 10 para a variável dependente "recebimento de kits básicos completos pelas SMS”. As variáveis independentes foram: proporção de unidades de saúde no município com gestão PAB e com gestão plena, população dos municípios, coeficiente de mortalidade infantil, proporção estimada da população coberta pelo Programa Saúde da Família (PSF), proporção de nascidos vivos de mães com mais de seis consultas no prénatal e IDH municipal. Considerou-se nessa análise o conglomerado e os pesos amostrais. $\mathrm{O}$ programa utilizado foi o Stata 7.0 (Stata Corp., College Station, Estados Unidos).

Foi desenvolvida uma abordagem qualitativa para descrever a atenção ao planejamento familiar e sua articulação com a estratégia de saúde da família. A definição da amostra para essa etapa da pesquisa foi feita em conjunto com a Área Técnica de Saúde da Mulher do Ministério da Saúde, com base nas proposições teóricas de Patton 6 sobre amostragem proposital. Levaramse em conta os seguintes critérios: resultados da etapa quantitativa, de maneira a incluir capitais em que a distribuição de métodos havia sido bem sucedida e outras em que isso não ocorrera; região geográfica, de maneira que houvesse uma capital de cada região do país; população, para incluir capitais mais e menos populosas; avaliação da Área Técnica sobre o estágio de organização da atenção à saúde da mulher e da implantação da estratégia de saúde da família, de forma a incluir capitais com e sem desempenho adequado nessas áreas. Devido à disponibilidade de recursos para realizar a pesquisa, as regiões Sul e Sudeste foram consideradas em conjunto. As capitais escolhidas foram Belém (Pará), Salvador (Bahia), Curitiba (Paraná) e Goiânia (Goiás), visitadas entre abril e maio de 2004.

Em cada cidade realizaram-se entrevistas semi-estruturadas com o(a) coordenador(a) de Saúde da Mulher, coordenador(a) do PSF, gerentes e profissionais de UBS e de equipes de saúde da família. Ao todo, obtiveram-se 99 entrevistas: 24 em Belém, 26 em Curitiba, 25 em Goiânia e 24 em Salvador.

Para a análise temática do conteúdo das entrevistas semi-estruturadas 11 , as categorias de análise (Tabela 1) foram definidas a partir dos objetivos do estudo, dos temas abordados nos roteiros e dos que emergiram das falas dos entrevistados.

O protocolo da pesquisa foi aprovado pelo Comitê de Ética em Pesquisa da Faculdade de Ciências Médicas, Universidade Estadual de Campinas (parecer n. 290/2002). Seguiram-se as determinações da Resolução 196/96 12. 
Categorias de análise utilizadas na etapa qualitativa da pesquisa.

\begin{tabular}{|c|c|}
\hline \multicolumn{2}{|c|}{ Categorias de análise } \\
\hline Aspectos estruturais & Aspectos operacionais \\
\hline Planejamento familiar no município & Atividades educativas \\
\hline Estratégia de saúde da família no município & Existência \\
\hline Relação saúde da família $\Leftrightarrow$ planejamento & Público-alvo/Demanda \\
\hline familiar $\Leftrightarrow$ Unidades Básicas de Saúde & Material educativo \\
\hline Priorização do planejamento familiar & Acesso \\
\hline Infertilidade como parte do planejamento familiar & Mulher adulta \\
\hline Provisão de insumos & Adolescente \\
\hline \multirow[t]{6}{*}{ Variedade/Disponibilidade de métodos anticoncepcionais } & Homem adulto \\
\hline & Esterilização feminina e masculina \\
\hline & Infertilidade \\
\hline & Processo de escolha de métodos anticoncepcionais \\
\hline & Anticoncepção de emergência \\
\hline & Demanda/Disponibilidade \\
\hline
\end{tabular}

\section{Resultados}

Somente $4 \%$ das SMS declararam não ter recebido nenhum método do kit básico, enquanto $66 \%$ referiram ter recebido kits completos. Cerca de um terço das SMS estimou que as quantidades recebidas durariam menos de dois meses. Dentre as UBS, $17 \%$ disseram não ter recebido nenhum método desse kit, e dentre as que receberam, cerca de um terço estimou que as quantidades durariam menos de dois meses (Tabela 2).

Mais de $90 \%$ das SMS receberam kits complementares completos. Um terço considerou que os DIU recebidos dariam para menos de dois meses, enquanto cerca da metade fez a mesma estimativa em relação ao hormonal injetável trimestral. Pouco mais de um terço das UBS declarou não ter recebido nenhum método desse kit, e quase $50 \%$ das que receberam estimaram que a quantidade duraria menos de dois meses (Tabela 3).

Cerca de $13 \%$ das SMS não referiram dificuldades para obter do Ministério da Saúde os métodos anticoncepcionais do kit básico. Entre as SMS com dificuldades, as mais freqüentes foram: "Ministério da Saúde demora em responder à demanda" (27,87\%), "fornece esses métodos em número insuficiente" (27,64\%), "foi a primeira vez que receberam métodos diretamente do Ministério" (27,45\%), "problemas de comunicação/não tinham contato com o Ministério/ não sabiam a quem pedir" (20,89\%) (Tabela 4).

A análise múltipla por regressão logística apontou que maior proporção de unidades em gestão plena, maior coeficiente de mortalidade infantil, IDH-M acima de 0,623 , maior população e maior proporção estimada da população coberta pela estratégia de saúde da família estavam associadas ao recebimento de kits básicos completos pelos municípios (Tabela 5).

Os principais resultados da etapa qualitativa da pesquisa, de acordo com as categorias de análise, foram os seguintes:

\section{Aspectos estruturais: planejamento familiar e saúde da família}

Nas capitais em que a saúde da família encontrava-se melhor implementada, verificou-se maior organização e efetividade das atividades de planejamento familiar. Embora os gestores municipais tenham enfatizado que essas atividades eram tão prioritárias quanto as demais da área de saúde da mulher, observou-se que, na prática, isto não acontecia. Entre os profissionais das UBS e das equipes de saúde da família, a maior ênfase continuava sendo o ciclo grávido-puerperal, e o planejamento familiar tendia a ocupar um plano secundário.

Os gestores municipais mencionaram a realização de capacitações em planejamento familiar tanto para profissionais de UBS quanto de equipes de saúde da família. Entretanto, os profissionais disseram que as capacitações, em geral, apenas incluíam o planejamento familiar dentro de outro tema, principalmente o pré-natal, e eles não se consideravam capacitados para atuarem em planejamento familiar. Em geral, os profissionais das equipes de saúde da fa- 
Resumo dos resultados do kit básico.

\begin{tabular}{lcc}
\hline & $\begin{array}{c}\text { Secretarias Municipais } \\
\text { de Saúde (\%) }\end{array}$ & $\begin{array}{c}\text { Unidades Básicas } \\
\text { de Saúde (\%) }\end{array}$ \\
\hline Recebimento de métodos anticoncepcionais* & & 28 \\
Kit completo & 66 & 17 \\
$\quad$ Nada & 4 & 45 \\
Pílula combinada & 83 & 31 \\
Nada & 8 & 47 \\
Minipílula & 86 & 44 \\
$\quad$ Nada & 13 & 47 \\
Preservativo & 80 & 21 \\
$\quad$ Nada & 7 & 31 \\
Duração (<2 meses) & & 38 \\
$\quad$ Pílula combinada & 33 & 34 \\
$\quad$ Minipílula & 37 & -
\end{tabular}

* As demais SMS/UBS receberam quantidades intermediárias de métodos anticoncepcionais. Fonte: Faúndes et al. 4.

Resumo dos resultados do kit complementar.

\begin{tabular}{|c|c|c|}
\hline & $\begin{array}{c}\text { Secretarias Municipais } \\
\text { de Saúde (\%) }\end{array}$ & $\begin{array}{c}\text { Unidades Básicas } \\
\text { de Saúde (\%) }\end{array}$ \\
\hline \multicolumn{3}{|c|}{ Recebimento de métodos anticoncepcionais* } \\
\hline Kit completo & 93 & 8 \\
\hline Nada & 2 & 36 \\
\hline DIU & 97 & 26 \\
\hline Nada & 2 & 44 \\
\hline Hormonal injetável & 96 & 11 \\
\hline Nada & 2 & 43 \\
\hline \multicolumn{3}{|l|}{ Duração (< 2 meses) } \\
\hline DIU & 33 & 25 \\
\hline Injetável & 49 & 29 \\
\hline Repasse total & 78 & - \\
\hline n & 97 & 116 \\
\hline
\end{tabular}

* As demais SMS/UBS receberam quantidades intermediárias de métodos anticoncepcionais. Fonte: Faúndes et al. 4.

mília não percebiam o planejamento familiar como parte da atenção básica, de caráter preventivo para a saúde da mulher e da criança.

Em duas capitais, as atividades de planejamento familiar não incluíam a infertilidade: não havia protocolo de atendimento nem sistema de referência. Nas outras duas capitais o aten- dimento à infertilidade era parte das ações em planejamento familiar, incluindo um sistema de referência das UBS ou equipes da saúde da família para serviços especializados. Porém, só em um desses municípios houve consistência entre as informações dos gestores e dos profissionais entrevistados a esse respeito. Na outra 
Dificuldades referidas pelos entrevistados das Secretarias Municipais de Saúde para obter do Ministério da Saúde os métodos anticoncepcionais do kit básico.

\begin{tabular}{|c|c|c|c|}
\hline & $\%$ & IC95\% & Deff \\
\hline \multicolumn{4}{|l|}{ Dificuldades $(n=491)$} \\
\hline Nenhuma dificuldade & 12,9 & $9,0-16,7$ & 1,68 \\
\hline Não souberam responder & 4,7 & $2,4-7,0$ & 1,44 \\
\hline \multicolumn{4}{|l|}{ Dificuldades $(n=366)$} \\
\hline Ministério da Saúde demora em responder à demanda/Pedem, mas nunca ou quase nunca recebem método & 27,8 & $22,5-33,2$ & 1,46 \\
\hline Ministério da Saúde fornece esses métodos em número insuficiente & 27,6 & $22,3-33,0$ & 1,51 \\
\hline Primeira vez que receberam método diretamente do Ministério da Saúde & 27,4 & $22,0-32,8$ & 1,53 \\
\hline $\begin{array}{l}\text { Problemas com comunicação/Não tinham contato com Ministério da Saúde/Não sabiam a quem pedir/ } \\
\text { Não sabiam da nova estratégia/Exigências de formulários }\end{array}$ & 20,8 & $15,9-25,8$ & 1,52 \\
\hline Dificuldades para obter métodos via Estado/Secretaria Estadual de Saúde/Regionais de Saúde & 17,9 & $13,3-22,5$ & 1,51 \\
\hline Ministério da Saúde não fornece regularmente & 15,0 & $10,9-19,1$ & 1,36 \\
\hline Outras dificuldades & 7,1 & $4,0-10,3$ & 1,53 \\
\hline Dificuldade com transporte/Município distante/Estrada ruim & 3,3 & $1,5-5,1$ & 1,01 \\
\hline Secretaria Municipal de Saúde/Município não tem recursos para comprar método & 3,1 & $1,2-5,1$ & 1,28 \\
\hline Não tem pessoal treinado/Município não está estruturado & 0,5 & $0,0-1,3$ & 0,94 \\
\hline
\end{tabular}

Fonte: Faúndes et al. 4.

Tabela 5

Variáveis associadas ao recebimento de kits completos distribuídos pelo Ministério da Saúde (análise por regressão logística múltipla, $\mathrm{n}=311$ ).

\begin{tabular}{|c|c|c|c|}
\hline Variável & Coeficiente & $\begin{array}{l}\text { Proporção estimada } \\
\text { (coeficiente) }\end{array}$ & $\mathrm{p}$ \\
\hline Proporção de unidades com gestão plena & 2,056 & 0,804 & 0,011 \\
\hline Coeficiente mortalidade infantil & 0,030 & 0,010 & 0,002 \\
\hline IDH municipal $(>0,623)^{*}$ & 0,976 & 0,391 & 0,013 \\
\hline População & $<0,001$ & $<0,001$ & 0,030 \\
\hline $\begin{array}{l}\text { Proporção estimada da população coberta } \\
\text { por equipes de saúde da família }\end{array}$ & 0,014 & 0,006 & 0,026 \\
\hline Constante & $-2,011$ & 0,645 & 0,002 \\
\hline
\end{tabular}

* Corte pela mediana.

Fonte: Faúndes et al. 4

capital, os profissionais pareciam não conhecer o sistema de referência mencionado pelos gestores.

Todos os entrevistados consideravam a provisão dos métodos anticoncepcionais como um dos pontos mais complicados das atividades de planejamento familiar. Em 2004, o suprimento de insumos era responsabilidade compartilhada entre municípios, estados e o nível federal. Apesar disso, em geral, os gestores e profissionais entendiam que a responsabilidade era só do Ministério da Saúde. Os municípios acabavam comprando métodos anticoncepcionais apenas pela demora, irregularidade e insuficiência dos insumos providos pelo Ministério.

Os gestores referiram dificuldades para planejar a compra de insumos por não saberem se e quando chegariam as remessas do Ministério da Saúde. Ressaltaram-se dificuldades de comunicação com o Ministério: por exemplo, não saber qual o melhor canal para pedir insumos, solicitar informações ou revisão dos quantita- 
tivos enviados ao município. Além disso, apontou-se também que o excesso de "burocracia" para compra de insumos em alguns municípios repercutia na falta de métodos anticoncepcionais nos serviços de saúde.

Profissionais das UBS e equipes de saúde da família relataram dificuldades de comunicação com o nível central em cada município, e que não sabiam como obter informações da coordenação municipal de saúde da mulher, solicitar suplementação de insumos etc.

De modo geral, os gestores e profissionais entendiam que a variedade de contraceptivos disponíveis nos municípios era satisfatória, porém a quantidade era insuficiente: a demanda era maior do que eram capazes de prover. Também apontaram dificuldades para fazer funcionar um sistema de referência e contra-referência das mulheres para outras UBS ou equipe de saúde da família em que um determinado método poderia ser obtido.

\section{Aspectos operacionais}

\section{- Atividades educativas}

A realização de atividades educativas em planejamento familiar, individuais ou grupais, foi referida em todas as capitais e aconteciam tanto nas UBS quanto em diferentes locais da comunidade. O público-alvo era principalmente mulheres. Os homens participavam eventualmente, quando estavam acompanhando as parceiras.

Foram poucas as referências a atividades educativas específicas para adolescentes, realizadas em escolas, e que enfocavam os métodos anticoncepcionais e diversos outros aspectos da saúde sexual e reprodutiva.

Em geral, nas UBS e equipes de saúde da família mencionou-se falta de material para ilustrar as palestras e distribuir às pessoas. Gestores e profissionais entrevistados consideravam que o Ministério da Saúde poderia contribuir para solucionar essa carência dando apoio para preparar esse tipo de material.

Referiu-se que as mulheres que solicitavam métodos anticoncepcionais recebiam informação completa sobre todos os contraceptivos disponíveis na rede pública, e não apenas sobre o método desejado. Essa informação era passada por uma enfermeira - individualmente ou em grupo, ou por um médico, durante a consulta. Os profissionais consideravam que as mulheres dificilmente mudavam de idéia, mesmo depois de receberem novas informações, pois chegavam até eles já com um método anticoncepcional em mente.
Parte dos profissionais considerava que as pessoas se inibiam e não compareciam a palestras exclusivamente sobre planejamento familiar. Era mais fácil abordar o assunto no âmbito de outros grupos educativos já existentes.

\section{- Acesso aos métodos contraceptivos}

\section{a) Mulheres adultas}

Em três capitais as mulheres tinham acesso aos métodos anticoncepcionais em livre demanda, sem necessidade de cadastro. Na outra capital, esse cadastramento era pré-requisito para receberem um método, porque a demanda era maior do que a disponibilidade de contraceptivos na rede pública.

Exceto em uma capital, os profissionais afirmaram que era difícil manter o suprimento de contraceptivos nas UBS ou equipes de saúde da família, e não era fácil saber para onde referir as mulheres para conseguirem o método anticoncepcional em uso. Com freqüência, davam uma receita para elas comprarem na farmácia. Situação semelhante ocorria quando as mulheres queriam iniciar o uso de um método que não estava disponível naquela UBS ou equipe de saúde da família.

Havia dificuldade das mulheres terem de passar por consulta médica a cada três meses, para obterem uma nova receita das pílulas anticoncepcionais, independentemente de terem alguma queixa ou não. Parte dos profissionais referiu que as(os) enfermeiras(os) transcreviam a receita quando não havia queixa que exigisse uma consulta médica. Porém, este não era um procedimento uniforme, mas cercado de controvérsias.

\section{b) Homens adultos}

As pessoas entrevistadas avaliaram que a presença dos homens em atividades de planejamento familiar era mínima, o que se atribuiu a questões culturais, que determinam que planejamento familiar "é coisa de mulher". Também mencionaram que o horário de funcionamento das UBS, em geral, não é adequado para os homens. Em nenhuma das capitais havia programa específico de saúde sexual e reprodutiva para homens.

\section{c) Adolescentes}

Segundo os gestores, não havia qualquer norma para restringir o acesso de adolescentes ao atendimento em planejamento familiar. Porém, havia profissionais de saúde que solicita- 
vam a presença de uma pessoa responsável pelo(a) adolescente. Alguns desses profissionais consideravam que estavam obedecendo a exigências legais. Outros referiram que só pediam a presença de um responsável quando entendiam que o(a) adolescente estava em situação de risco, como, por exemplo, quando suspeitavam de abuso sexual.

Gestores e profissionais referiram pequena procura espontânea de adolescentes pelos serviços de saúde. As adolescentes tendiam a procurar as UBS ou equipes de saúde da família quando já estavam grávidas. Em todas as capitais visitadas havia preocupação com os índices de gravidez na adolescência e grande ênfase quanto à necessidade de atuar para evitá-la. Para os adolescentes homens, centravam-se esforços na prevenção de doenças sexualmente transmissíveis.

Referiram-se várias tentativas de "captar" adolescentes nas comunidades, especialmente através dos agentes comunitários de saúde e das equipes de saúde da família. Uma das estratégias mais usadas eram as palestras nas escolas, feitas pelos profissionais de saúde. Apenas em uma capital havia programa específico para adolescentes, apoiado por cartilhas educativas, e se disponibilizavam preservativos nas escolas.

\section{- Atendimento à demanda pela esterilização cirúrgica feminina e masculina}

De modo geral, os gestores entendiam que havia demanda reprimida pela esterilização cirúrgica, acumulada desde a entrada em vigor da regulamentação legal. A demanda pela vasectomia era menor do que pela laqueadura, embora fosse oferecida muitas vezes de forma intencional aos casais cuja mulher solicitava a laqueadura.

Em duas capitais existiam serviços credenciados para realizar a laqueadura e a vasectomia, com critérios e procedimentos definidos: oferta de métodos reversíveis, atividades educativas, encaminhamento. Em uma dessas cidades, porém, os profissionais não revelaram clareza quanto a esses procedimentos, e referiram não ter como acompanhar o atendimento às pessoas que solicitavam as cirurgias.

Em duas capitais não havia serviços credenciados, porém os entrevistados reconheciam que a laqueadura era realizada mediante "arranjos pessoais” entre mulheres e médicos.

\section{- Anticoncepção de emergência}

Em três capitais os gestores referiram que a pílula de anticoncepção de emergência (PAE) es- tava disponível nas UBS e equipes de saúde da família, porém, havia profissionais desses serviços que não sabiam disso. Em uma capital a PAE não estava disponível na rede pública no momento da pesquisa. Anteriormente, já estivera em algumas UBS.

Em geral, referiu-se pequena demanda pela PAE. Em uma capital, os profissionais de saúde disseram que, quando houve demanda, foi de adolescentes. Ao mesmo tempo, nas quatro localidades, a existência do método não era - ou era pouco - divulgada à população.

Gestores e profissionais consideravam que a PAE só poderia ser dispensada mediante consulta médica. Havia preocupação de não "ficar distribuindo por aí, porque é uma coisa de emergência". Em uma capital a tendência foi afirmar que a PAE só podia ser utilizada nos casos de violência sexual, e sempre prescrita por um médico.

Nas quatro capitais havia cuidados especiais para armazenar e dispensar a PAE. Em alguns serviços, o medicamento estava sob a responsabilidade do(a) coordenador(a), que era a única pessoa que poderia entregá-lo, mediante prescrição médica.

\section{Discussão}

Nossos resultados confirmam que a atenção ao planejamento familiar no Brasil continua a ser marcada pela indisponibilidade de métodos anticoncepcionais nos serviços públicos de saúde, e pela capacitação desigual e insuficiente dos profissionais para atuarem nessa área.

As ações em planejamento familiar, previstas no PAISM como parte da atenção integral à saúde da mulher 1,13, incluem-se na atenção básica, de acordo com o modelo de atenção à saúde adotado no Brasil desde a criação do SUS, passando pela estratégia de saúde da família 14 . Percebe-se, entretanto, que a assistência ao planejamento familiar nos municípios está ainda distante de um patamar satisfatório. As ações de planejamento familiar, com freqüência, são executadas de forma isolada e não priorizadas como os demais componentes da atenção básica.

Nesta pesquisa ficou claro que os profissionais das equipes da saúde da família não entendiam o planejamento familiar como parte da atenção básica e não se consideravam capacitados para prestar assistência nessa área, confirmando resultados de uma avaliação do processo de implantação do PSF, que indicaram que apenas cerca de um terço das equipes de saúde da família tinha médicos(as) capacita- 
dos(as) em saúde da mulher, e $40 \%$ tinham enfermeiros(as) capacitados(as) 15.

Também se evidenciou neste estudo a dificuldade de funcionamento de um sistema de referência e contra-referência para as ações de planejamento familiar, fundamental para racionalizar a utilização dos recursos disponíveis. Embora esse sistema tenha sido mencionado pelos gestores entrevistados nas quatro capitais, os profissionais das UBS e das equipes de saúde da família não pareciam familiarizados e/ou não davam crédito ao mesmo.

Chama atenção também que em duas capitais visitadas nem havia serviços credenciados para realizar laqueadura e vasectomia, o que deixava as portas abertas para que persistissem as distorções na obtenção dessas formas de contracepção, tão discutidas na literatura sobre planejamento familiar no Brasil. Nossos resultados concordam com outros estudos $16,17,18,19$, 20 em que o atendimento da demanda pela esterilização cirúrgica ainda é insuficiente no SUS, apesar de estar regulamentado desde 1997.

Em relação às ações voltadas a adolescentes, ao mesmo tempo em que se observou grande ênfase sobre a necessidade desse grupo conhecer e utilizar métodos anticoncepcionais para evitar a gravidez nessa faixa etária, ficou evidente a pouca organização do atendimento em planejamento familiar para adolescentes. Também se verificou que, em geral, os profissionais de saúde vivenciavam conflitos em sua prática diária quanto à autonomia dos(as) adolescentes para assumirem a responsabilidade de seu atendimento, e tomarem decisões sobre o uso de métodos anticoncepcionais, embora o Ministério da Saúde 21 venha enfatizando os direitos dos(as) adolescentes à preservação do sigilo e ao consentimento informado, bem como a necessidade de abordagens adequadas a essa etapa da vida. Porém, é preciso lembrar que orientar e atender adolescentes em questões de anticoncepção, bem como para a prevenção de doenças de transmissão sexual e AIDS, ultrapassam os aspectos de competência técnica, pois envolve o campo dos valores, especialmente no que se refere ao exercício da sexuali- dade. Portanto, a capacitação dos profissionais para essa tarefa deve incluir a discussão sobre o lugar da sexualidade no processo de autonomização juvenil, que ainda hoje é fortemente marcado pela hierarquia de gênero 22,23 .

Esta pesquisa reitera a existência de problemas crônicos quanto a quem corresponde comprar, e como assegurar que os métodos anticoncepcionais estejam disponíveis nas UBS e equipes de saúde da família em quantidade e variedade adequadas. A centralização da compra pelo Ministério da Saúde voltou a ser proposta em 2005, mas essa medida não garante a disponibilidade de métodos anticoncepcionais nos municípios. Isso porque são conhecidos os tropeços que o Ministério tem enfrentado para comprar e distribuir métodos anticoncepcionais 3 . Além disso, ainda quando a responsabilidade deveria ser compartilhada pelos três níveis de governo, a idéia que prevalecia era de que a provisão de métodos anticoncepcionais era responsabilidade do Ministério. Portanto, será necessária a adequada compreensão dos municípios acerca dessa proposta, e a definição do Ministério sobre como e em qual prazo pretende avançar na provisão dos insumos contraceptivos, para evitar que alguns municípios entendam que, desde agora, não precisam destinar recursos para a aquisição de anticoncepcionais.

Entretanto, parece-nos fundamental frisar que, mesmo que o Ministério da Saúde consiga enviar os insumos contraceptivos aos municípios com regularidade e em quantidades adequadas, isso não garantirá que a atenção ao planejamento familiar alcance a qualidade e a eficácia desejadas para permitir aos cidadãos brasileiros exercerem o direito de decidir quando e quantos filhos querem ter. Isso é necessário, porém não suficiente, a menos que os municípios tratem as ações em planejamento familiar, de fato, como parte da atenção básica. É preciso que o processo de humanização da atenção também alcance o planejamento familiar, no que se refere à incorporação de princípios relativos aos direitos humanos e à bioética na atenção brindada à população. 


\section{Resumo}

Realizou-se estudo com uma primeira etapa descritiva, de corte transversal, e segunda etapa qualitativa, de estudo de casos. Avaliou-se a disponibilidade de métodos anticoncepcionais nas Unidades Básicas de saúde de municípios brasileiros, e características da atenção ao planejamento familiar e a articulação com a estratégia de saúde da família. Inicialmente, foram aplicados, por telefone, questionários sobre o recebimento recente de métodos anticoncepcionais, em municípios selecionados a partir de um plano complexo de amostragem. Na etapa qualitativa foram selecionados propositalmente e visitados quatro municípios, onde se realizaram observação e entrevistas semi-estruturadas com gestores e profissionais de saúde. Procedeu-se a análise estatística descritiva e regressão logística múltipla dos dados quantitativos. Para os qualitativos adotou-se a técnica de análise temática do conteúdo. Verificou-se que as ações de planejamento familiar, com freqüencia, são executadas de forma isolada e que os profissionais das equipes de saúde da família não entendiam o planejamento familiar como parte da atenção básica e não se consideravam capacitados para prestar assistência nessa área. A atenção a planejamento familiar continua a ser marcada pela indisponibilidade de métodos anticoncepcionais nos serviços públicos de saúde.

Planejamento Familiar; Anticoncepção; Atenção Básica à Saúde; Saúde da Família

\section{Colaboradores}

A. Faúndes, M. B. Mello, M. H. Sousa e M. J. D. Osis participaram de todo o desenvolvimento da pesquisa, incluindo a análise de dados que deu origem ao artigo. M. Y. Makuch e M. J. O. Araújo participaram do desenho, desenvolvimento e análise de dados do componente qualitativo do estudo. A. Faúndes e M. J. D. Osis prepararam o manuscrito, que foi revisado por cada um dos demais co-autores.

\section{Agradecimentos}

Os autores agradecem à Dra. Tânia Lago pela contribuição no desenvolvimento da etapa quantitativa da pesquisa realizada, e à Dra. Isa Paula Hamuche Abreu pela cooperação na etapa qualitativa.

\section{Referências}

1. Costa AM. Desenvolvimento e implementação do PAISM no Brasil. In: Giffin K, Costa SH, organizadoras. Questões da saúde reprodutiva. Rio de Janeiro: Editora Fiocruz; 1999. p. 319-55.

2. Pinotti JA, Faúndes A, Hardy E, Rebelo-Simões I, Osis MJD, Souza TR, et al. Avaliação da assistência ginecológica no Estado de São Paulo. Rev Ginecol Obst 1990; 1:7-21.

3. Área Técnica de Saúde da Mulher, Ministério da Saúde. Direitos sexuais e direitos reprodutivos: uma prioridade do governo. Brasília: Ministério da Saúde; 2005.

4. Faúndes A, Osis MJD, Sousa MH, Mello MB. Avaliação da estratégia de distribuição de métodos anticoncepcionais no Brasil pelo Ministério da Saúde. Relatório Técnico. Campinas: Centro de Pesquisas em Saúde Reprodutiva de Campinas; 2002.

5. Faúndes A, Mello MB, Osis MJD, Makuch MY. Avaliação da estratégia de distribuição de métodos anticoncepcionais no Brasil pelo Ministério da Saúde - estudo de casos. Relatório Técnico. Campinas: Centro de Pesquisas em Saúde Reprodutiva de Campinas; 2004.

6. Patton MQ. Qualitative evaluation and research methods. 2nd Ed. London: Sage Publications; 1990.

7. Altman DG. Practical statistics for medical research. New York: Chapman \& Hall; 1999.

8. Kish L. Survey sampling. New York: John Wiley \& Sons; 1965.

9. Sousa MH, Silva NN. Estimativas obtidas de um levantamento complexo. Rev Saúde Pública 2003; 37:662-70.

10. Hosmer DW, Lemeshow S. Applied logistic regression. New York: John Wiley \& Sons; 1989.

11. Minayo MCS. O desafio do conhecimento: pesquisa qualitativa em saúde. 5a Ed. São Paulo: Editora Hucitec/Rio de Janeiro: ABRASCO; 1998.

12. Ministério da Saúde/Conselho Nacional de Saúde. Resolução 196/96 sobre pesquisa envolvendo seres humanos. Bioética 1996; 4:15-25.

13. Osis MJD. PAISM: um marco na abordagem da saúde reprodutiva no Brasil. Cad Saúde Pública 1998; 14 Suppl 1:25-32.

14. Souza HM, Sampaio LFR. Atenção básica: políticas e diretrizes no Brasil atual. Brasília: Ministério da Saúde; 2002.

15. Departamento de Atenção Básica, Secretaria de Atenção à Saúde, Ministério da Saúde. Avaliação normativa do Programa Saúde da Família no Brasil: monitoramento da implantação e funcionamento das equipes de saúde da família - 2001/ 2002. Brasília: Ministério da Saúde; 2004.

16. Berquó E, Cavenaghi S. Direitos reprodutivos de mulheres e homens face à nova legislação sobre esterilização voluntária. Cad Saúde Pública 2003; 19 Suppl 2:S441-53.

17. Luiz OL, Citeli MT. Esterilização cirúrgica voluntária na Região Metropolitana de São Paulo: organização e oferta de serviços, 1999. http://www.redesaude.org.br/jornal/html/body_jr21-olinda. html (acessado em 12/Nov/2003).

18. Potter JE, Pérpetuo IHO, Berquó E, Hopkins K, Leal OF, Formiga MC, et al. Frustrated demand 
for postpartum female sterilization in Brazil. Contraception 2003; 67:385-90.

19. Vieira EM, Ford NJ. Oferta da esterilização feminina em Ribeirão Preto, São Paulo, Brasil. Cad Saúde Pública 2004; 20:1201-10.

20. Osis MJD, Carvalho LEC, Manfrinatti MB, Bento SF. Atendimento à demanda pela esterilização cirúrgica voluntária na rede pública da Região Metropolitana de Campinas. Relatório Parcial de Pesquisa Apresentado à Fundação de Amparo à Pesquisa do Estado de São Paulo (FAPESP). Campinas: Centro de Pesquisas em Saúde Reprodutiva de Campinas; 2005.
21. Área de Saúde do Adolescente e do Jovem, Secretaria de Atenção à Saúde, Ministério da Saúde. Marco legal: saúde, um direito de adolescente. Brasília: Ministério da Saúde; 2005.

22. Heilborn ML, Aquino EML, Knauth DR. Juventude, sexualidade e reprodução. Cad Saúde Pública 2006; 22:1362-3.

23. Brandão ER, Heilborn ML. Sexualidade e gravidez na adolescência entre jovens de camadas médias no RJ, Brasil. Cad Saúde Pública 2006; 22:1421-30.

Recebido em 04/Mai/2006 Aprovado em 27/Jul/2006 